\title{
Effects of remifentanil on hemodynamics, liver function and ICAM-1 expression in liver cancer patients undergoing surgery
}

\author{
QI JIANG ${ }^{1 *}$, XIULING SONG ${ }^{2 *}$, ZHENG CHEN $^{3}$, CONGHUI WANG $^{1}$ and HUIYU LUO ${ }^{1}$ \\ Departments of ${ }^{1}$ Anesthesiology and ${ }^{2}$ Endocrinology, Xiangyang No. 1 People's Hospital, Hubei University \\ of Medicine, Xiangyang, Hubei 441000; ${ }^{3}$ Department of Hepatobiliary Surgery, Guangdong Province \\ Key Laboratory of Malignant Tumor Epigenetics and Gene Regulation, Research Center of Medicine, \\ Sun Yat-Sen Memorial Hospital, Sun Yat-Sen University, Guangzhou, Guangdong 510275, P.R. China
}

Received February 3, 2017; Accepted May 2, 2017

DOI: $10.3892 / 01.2017 .6247$

\begin{abstract}
The objective of the present study was to investigate the effects of remifentanil on hemodynamics, liver function, and expression of intercellular adhesion molecule-1 (ICAM-1) in patients with liver cancer undergoing surgery. A total of 60 patients who underwent liver cancer resection in The First People's Hospital of Xiangyang, Hubei University of Medicine from January 2014 to January 2016 were selected, including 33 males and 27 females, with an average age of $54.12 \pm 4.77$ years. Patients were randomly divided into the control group and experimental group ( $\mathrm{n}=30$ each). The control group and experimental group were anesthetized with propofol/isoflurane and remifentanil/propofol, respectively. In addition to general parameters, the following parameters were analyzed: mean systolic blood pressure and mean diastolic blood pressure were obtained before treatment, during anesthesia induction and intubation, during blockade of traction reflexes in surgery, and before extubation at the end of surgery. The recovery time from anesthesia withdrawal to spontaneous breathing, time of eye opening, time of extubation, and level of consciousness were recorded. Liver expression of ICAM-1 was measured with SABC staining, and the expression of aspartate aminotransferase (AST), alanine aminotransferase (ALT), and total bilirubin (TBIL) were recorded on the first, third, fifth, and seventh day after surgery. According to hemodynamic parameters, patients in the experimental group experienced a more stable condition than patients in the control group $(\mathrm{P}<0.05)$. In addition, the recovery time of the experimental group was shorter than that of the control group $(\mathrm{P}<0.05)$. Markers of liver function
\end{abstract}

Correspondence to: Dr Huiyu Luo, Department of Anesthesiology, Xiangyang No. 1 People's Hospital, Hubei University of Medicine, 15 Jiefang Road, Xiangyang, Hubei 441000, P.R. China

E-mail: h715zf@163.com

${ }^{*}$ Contributed equally

Key words: remifentanil, ischemia-reperfusion injury, hemodynamics, liver function
(AST, ALT and TBIL) of the two groups after surgery were higher than those before surgery, and the increases of the experimental group were significantly lower than those of the control group. ICAM-1 expression in the experimental group was significantly lower than in the control group $(\mathrm{P}<0.05)$. In conclusion, anesthesia with remifentanil better maintained the stability of hemodynamics, played a protective role against hepatic ischemia-reperfusion injury, and reduced ICAM-1 expression.

\section{Introduction}

At present, resection is an important treatment option for liver cancer. However, intraoperative liver damage is often inevitable. Ischemia-reperfusion injury in liver surgery is a common pathophysiological consequence in patients (1). Patients with liver cancer may already experience a certain degree of liver dysfunction before surgery. Therefore, choosing an appropriate anesthetic is key for minimizing further damage to the liver during this type of surgery.

On the surface of hepatocytes, hepatic sinusoidal endothelial cells and vascular endothelial cells express various adhesion molecules, among which intercellular adhesion molecule-1 (ICAM-1) is primarily expressed in liver tissue. The expression of ICAM-1 can serve as a biomarker of the extent of liver damage, where higher expression indicates more severe liver damage (2). In the present study, remifentanil was used for general anesthesia of patients undergoing liver cancer surgery, to investigate the effects of remifentanil on liver function and ICAM-1 expression, as well as blood pressure variation in patients. The findings may serve as a reference for selecting appropriate anesthesia during surgery.

\section{Patients and methods}

Patients. A total of 60 patients who underwent liver cancer resection in The First People's Hospital of Xiangyang, Hubei University of Medicine (Xiangyang, China) were randomly selected from January 2014 to January 2016, including 33 males and 27 females with average age of $54.12 \pm 4.77$ years. All selected patients were designated as class I or class II according to the American Society of Anesthesiologists 
Physical Status classification system, and Class A according to the Child-Pugh classification of liver disease. The patients were randomly divided into the control group and experimental group, with 30 cases in each group. There were 17 males and 13 females in the control group, with average age of $54.87 \pm 3.76$ years, and 15 males and 15 females in the experimental group, with an average age of $53.43 \pm 4.12$ years. There were no significant differences in preoperative liver function tests between patients, and surgery was completed by the same group of physicians in The First People's Hospital of Xiangyang, Hubei University of Medicine. Patients had no history of mental disease, neurological disease, or serious cardiovascular disease; no recent administration of narcotic analgesics and sedative hypnotics; and no history of using drugs that can cause liver damage, such as benzodiazepines and opioids. This study was approved by the Ethics Committee of Xiangyang No. 1 People's Hospital. Signed written informed consents were obtained from all participants before the study.

Anesthesia. Patients in the control group were anesthetized with propofol/isoflurane, whereas patients in the experimental group were administered remifentanil/propofol (Yangze Pharma, Taizhou, China). Patients were connected to the electrocardiogram monitor, and received oxygen flow through a face mask after entering the operating room. Physiological indicators such as blood pressure, heart rate (HR), and finger oxygen saturation were monitored. After establishing the upper extremity venous access, a catheter was placed in the right internal jugular vein for measuring the central venous pressure while keeping the venous access open. Anesthetics such as remifentanil, propofol, and midazolam (Yangze Pharma) were administered to patients at the dose of $4 \mu \mathrm{g} / \mathrm{kg}, 2.5 \mathrm{mg} / \mathrm{kg}$, and $0.05 \mathrm{mg} / \mathrm{kg}$, respectively, for anesthesia induction before anesthesia to facilitate intubation. Patients in the control group were anesthetized with propofol/isoflurane. Following the protocol, propofol was administered intravenously at a dose of $6.5-9.0 \mathrm{mg} / \mathrm{kg} / \mathrm{h}$, and $2-3 \%$ isoflurane was simultaneously administered by inhalation. The administration of isoflurane and propofol was stopped 15 and $5 \mathrm{~min}$, respectively, before surgery was finished. During surgery, the concentration of isoflurane was adjusted in time in accordance with the depth of anesthesia and changes of patient hemodynamic parameters. Patients in the experimental group were anesthetized with remifentanil/propofol at a dose of $0.3-0.5 \mu \mathrm{g} / \mathrm{kg} / \mathrm{min}$ and $7-10 \mathrm{mg} / \mathrm{kg} / \mathrm{h}$, respectively. The doses of remifentanil and propofol were adjusted according to hemodynamic changes during surgery. Infusion of remifentanil and propofol was stopped $5 \mathrm{~min}$ before surgery was finished. Patients in the two groups were extubated after wake-up, recovery of spontaneous breathing, and activation of cough and swallowing reflexes. Finally, patients were sent to the intensive care unit.

ICAM-1 measurement. Hepatic blood flow was controlled during surgery in patients in the two groups when liver tissues were harvested. The obtained liver tissues were marked and stored in liquid nitrogen. The SABC staining technique was used to determine the expression of ICAM-1 in liver tissue. Briefly, samples were first prepared and cut into $5 \mu \mathrm{m}$ paraffin-embedded sections. The sections were then affixed on microscope glass slides using an adhesive. After drying, the sections were dewaxed and hydrated. After endogenous enzymes were inactivated at $20^{\circ} \mathrm{C}$ for $10 \mathrm{~min}$, the sections were washed three times with distilled water, and then immersed in citrate buffer. The buffer was heated until it boiled, and was then left to stand for $10 \mathrm{~min}$ without heating. After repeating the heating/cooling cycle two times, the sections were rinsed two times with PBS, followed by the addition of antigen retrieval buffer to expose the antigen. The sections were then rinsed two times with PBS, followed by addition of BSA blocking buffer, and incubation for $20 \mathrm{~min}$. Rabbit anti-rat ICAM-1 monoclonal antibody was added and incubated at $4^{\circ} \mathrm{C}$. After warming the sections to room temperature, they were rinsed with PBS, followed by the addition of SABC reagent, and incubation. The sections were placed in a kit and developed at $20^{\circ} \mathrm{C}$, followed by washing, counterstaining, dehydration, clearing, and mounting. The sections were then observed under a light microscope (BX-42; Olympus, Tokyo, Japan). ICAM-1 antibody, DAB chromogenic reagent, and other immunohistochemistry reagents were purchased from Wuhan Boster Biological Technology, Ltd. (Wuhan, China).

Observational indicators. The general parameters of patients (preoperative) were measured and recorded, such as sex, age, mean HR, mean arterial pressure (MAP), and liver function markers including aspartate aminotransferase (AST), alanine aminotransferase (ALT), and total bilirubin (TBIL). The mean systolic blood pressure (SBP) and mean diastolic blood pressure (DBP) were obtained before treatment, during anesthesia induction and intubation, during blockade of traction reflexes in surgery, and before extubation at the end of surgery. The recovery time from anesthesia withdrawal to spontaneous breathing, time of eye opening, time of extubation, as well as the level of consciousness were recorded. The level of consciousness was graded according to the Observer's Assessment of Alertness/Sedation Scale. Patients received a score of 5 if they replied readily to name call and were fully awake, a score of 4 if they replied sluggishly to name call with a slow speech rate, a score of 3 if they replied only to loud name call with blurred speech and dull eyes, a score of 2 if they did not reply to name call and only responded to mild prodding or shaking, and a score of 1 if they were in a lethargic state and did not respond to mild prodding and shaking. In addition, the levels of liver function markers, AST, ALT, and TBIL were measured in all patients at the first (D1), third (D3), fifth (D5), and seventh (D7) day after surgery.

Statistical analysis. SPSS 19.0 statistical software was used for data analysis (IBM SPSS, Armonk, NY, USA). Numerical data are presented as mean \pm standard deviation. A Chi-squared $\left(\chi^{2}\right)$ test was used for categorical data. The t-test for two independent samples was used for intergroup comparisons. Repeated measures analysis of variance was employed for intragroup comparisons. $\mathrm{P}<0.05$ was considered to be statistically significant.

\section{Results}

General parameters (preoperative). There were no significant differences in sex, age, HR, MAP, AST, ALT, or TBIL between the control group and experimental group ( $\mathrm{P}>0.05$; Table I). 
Table I. General parameters of patients (preoperative).

\begin{tabular}{lcccccc}
\hline Group & $\begin{array}{c}\text { Sex } \\
(\text { male/female })\end{array}$ & $\begin{array}{c}\text { Age } \\
(\text { years })\end{array}$ & $\begin{array}{c}\text { HR } \\
(\text { per min })\end{array}$ & $\begin{array}{c}\text { MAP } \\
(\mathrm{mm} \mathrm{Hg})\end{array}$ & $\begin{array}{c}\text { AST } \\
(\mu \mathrm{mol} / \mathrm{l})\end{array}$ & $\begin{array}{c}\text { ALT } \\
(\mu \mathrm{mol} / \mathrm{l})\end{array}$ \\
\hline Control & $17 / 13$ & $54.87 \pm 3.76$ & $82.95 \pm 7.28$ & $87.54 \pm 10.29$ & $41.28 \pm 11.39$ & $47.13 \pm 12.84$ \\
$(\mu \mathrm{mol} / \mathrm{l})$
\end{tabular}

HR, heart rate; MAP, mean arterial pressure; AST, aspartate aminotransferase; ALT, alanine aminotransferase; TBIL, total bilirubin.

Table II. Hemodynamic data of the two groups at different time points.

\begin{tabular}{|c|c|c|c|c|c|}
\hline Group & $\begin{array}{l}\text { Blood } \\
\text { pressure }\end{array}$ & $\begin{array}{l}\text { Before } \\
\text { surgery }\end{array}$ & $\begin{array}{l}\text { During anesthesia } \\
\text { induction and } \\
\text { intubation }\end{array}$ & $\begin{array}{l}\text { During blockade of } \\
\text { traction reflexes } \\
\text { in surgery }\end{array}$ & $\begin{array}{l}\text { Before extubation } \\
\text { at the end of } \\
\text { surgery }\end{array}$ \\
\hline \multirow[t]{2}{*}{ Control } & $\mathrm{SBP}(\mathrm{mm} \mathrm{Hg})$ & $132.25 \pm 15.43$ & $119.95 \pm 14.52$ & $150.37 \pm 17.27^{\mathrm{a}, \mathrm{c}}$ & $145.63 \pm 12.54^{\mathrm{a}, \mathrm{c}}$ \\
\hline & $\mathrm{DBP}($ per min) & $78.47 \pm 10.52$ & $72.54 \pm 7.28$ & $88.76 \pm 11.75^{\mathrm{a}, \mathrm{c}}$ & $86.49 \pm 10.17^{\mathrm{a}, \mathrm{c}}$ \\
\hline \multirow[t]{2}{*}{ Experimental } & SBP (mm Hg) & $133.78 \pm 14.53$ & $122.14 \pm 13.43$ & $124.07 \pm 13.12^{\mathrm{b}, \mathrm{c}}$ & $123.82 \pm 12.89^{\mathrm{b}, \mathrm{c}}$ \\
\hline & $\mathrm{DBP}$ (per min) & $77.68 \pm 11.17$ & $72.88 \pm 8.15$ & $73.96 \pm 9.82^{\mathrm{b}, \mathrm{c}}$ & $72.19 \pm 9.48^{\mathrm{b}, \mathrm{c}}$ \\
\hline
\end{tabular}

${ }^{\mathrm{a}} \mathrm{P}<0.05$, compared with data during anesthesia induction and intubation within the group; ${ }^{\mathrm{b}} \mathrm{P}>0.05$, compared with data during anesthesia induction and intubation within the group; ${ }^{~} \mathrm{P}<0.05$, compared with data in the other group at the same time point. SBP, systolic blood pressure; DBP, diastolic blood pressure.

Table III. Recovery data of the two groups.

\begin{tabular}{|c|c|c|c|c|}
\hline Group & $\begin{array}{c}\text { Recovery time of } \\
\text { spontaneous breathing }\end{array}$ & $\begin{array}{c}\text { Time of } \\
\text { eye opening }\end{array}$ & Extubation time & $\begin{array}{c}\text { Level of } \\
\text { consciousness }\end{array}$ \\
\hline Control & $8.81 \pm 3.51^{\mathrm{a}}$ & $19.53 \pm 4.69^{\mathrm{a}}$ & $21.27 \pm 5.83^{\mathrm{a}}$ & $3.96 \pm 0.67^{\mathrm{a}}$ \\
\hline Experimental & $5.42 \pm 2.64$ & $11.66 \pm 3.92$ & $13.73 \pm 4.38$ & $4.48 \pm 0.31$ \\
\hline
\end{tabular}

${ }^{a} \mathrm{P}<0.05$, compared with the experimental group.

Hemodynamic data. In the control group, SBP and DBP were significantly higher during blockade of traction reflexes in surgery and before extubation at the end of surgery compared with the values during anesthesia induction and intubation $(\mathrm{P}<0.05)$. However, in the experimental group, SBP and DBP were comparable at these time points $(\mathrm{P}>0.05)$. Comparing the two groups, SBP and DBP during blockade of traction reflexes in surgery and before extubation at the end of surgery were lower in the experimental group than in the control group $(\mathrm{P}<0.05$; Table II $)$.

Recovery time. In the comparison of recovery time of spontaneous breathing, time of eye opening, extubation time, and level of consciousness (Table III), the experimental group was better than the control group, and the differences were statistically significant $(\mathrm{P}<0.05)$.

Liver function. The levels of the liver function markers (AST, ALT, and TBIL) of the two groups of patients were significantly higher after surgery compared with those before surgery $(\mathrm{P}<0.05)$. The levels of AST, ALT, and TBIL on the first, third, and fifth day after surgery were higher in the control group than in the experimental group, and the differences were statistically significant $(\mathrm{P}<0.05)$. On the seventh day, the levels of the three markers returned to normal in both groups, and there were no significant differences between the two groups (Table IV).

ICAM-1 expression. The expression of ICAM-1 was $54.31 \%$ in the control group, which was significantly higher than in the experimental group $(37.66 \%)(\mathrm{P}<0.05$; Fig. 1).

\section{Discussion}

According to global statistics, liver cancer ranked third among cancer deaths in 2002 (3). Primary liver cancer is not only a common malignant tumor, but also one of the leading causes of death. According to year 2000 statistics, the number 
Table IV. The levels of liver function markers.

\begin{tabular}{|c|c|c|c|c|c|c|}
\hline Group & Marker & D0 & D1 & D3 & D5 & D7 \\
\hline \multirow[t]{3}{*}{ Control } & AST & $41.28 \pm 11.39$ & $188.46 \pm 29.19^{\mathrm{a}, \mathrm{c}}$ & $152.93 \pm 34.62^{\mathrm{a}, \mathrm{c}}$ & $90.63 \pm 23.64^{\mathrm{a}, \mathrm{c}}$ & $47.41 \pm 13.64$ \\
\hline & ALT & $47.13 \pm 12.84$ & $196.11 \pm 32.75^{\mathrm{a}, \mathrm{c}}$ & $160.37 \pm 34.51^{\mathrm{a}, \mathrm{c}}$ & $99.82 \pm 25.77^{\mathrm{a}, \mathrm{c}}$ & $49.63 \pm 14.96$ \\
\hline & TBIL & $15.24 \pm 7.93$ & $19.81 \pm 8.1^{\mathrm{a}, \mathrm{c}}$ & $24.14 \pm 8.69^{\mathrm{a}, \mathrm{c}}$ & $27.59 \pm 10.67^{\mathrm{a}, \mathrm{c}}$ & $16.17 \pm 7.81$ \\
\hline \multirow[t]{3}{*}{ Experimental } & AST & $39.52 \pm 12.63$ & $154.82 \pm 26.39^{\mathrm{b}, \mathrm{c}}$ & $129.86 \pm 25.19^{b, c}$ & $72.47 \pm 19.78^{b, c}$ & $40.42 \pm 10.56$ \\
\hline & ALT & $45.21 \pm 11.83$ & $161.14 \pm 31.46^{\mathrm{b}, \mathrm{c}}$ & $131.43 \pm 30.31^{\mathrm{b}, \mathrm{c}}$ & $75.97 \pm 20.73^{b, c}$ & $42.55 \pm 11.87$ \\
\hline & TBIL & $15.87 \pm 8.44$ & $17.15 \pm 7.88^{b, c}$ & $22.97 \pm 8.13^{b, c}$ & $18.77 \pm 9.64^{\mathrm{b}, \mathrm{c}}$ & $15.93 \pm 8.11$ \\
\hline
\end{tabular}

${ }^{\mathrm{a}} \mathrm{P}<0.05$, compared with $\mathrm{D} 0$ within the control group; ${ }^{\mathrm{b}} \mathrm{P}<0.05$, compared with $\mathrm{D} 0$ within the experimental group; ${ }^{\mathrm{c}} \mathrm{P}<0.05$, compared with the same day in the other group. AST, aspartate aminotransferase; ALT, alanine aminotransferase; TBIL, total bilirubin.

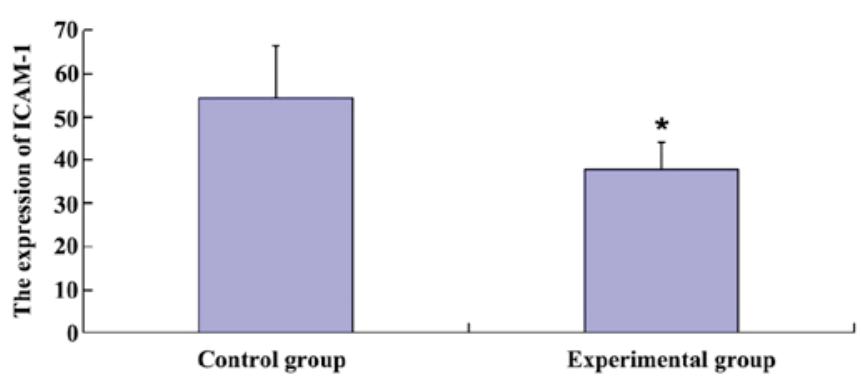

Figure 1. ICAM-1 expression in the two groups by SABC staining. ICAM-1, intercellular adhesion molecule-1. ${ }^{*} \mathrm{P}<0.05$.

of cases of liver cancer in China accounted for $55 \%$ of the global case number, indicating that China has become a country with high incidence of liver cancer. Moreover, the number of cases is increasing. The number of new cases each year has reached approximately 350,000 (4). Presently, there are effective ways to treat liver cancer, including surgery, radiotherapy and chemotherapy, liver transplantation, biological therapy, and Chinese medicine (5). Surgery with resection offers the only chance to cure liver cancer, thus partial hepatectomy is currently the preferred method of treatment for liver cancer (6). Operative blood loss is a major risk factor associated with operative mortality after partial hepatectomy. For effective bleeding control, temporary occlusion of hepatopetal blood flow is highly necessary. However, this procedure may result in ischemia-reperfusion injury of hepatocytes. For patients with poor liver function, reperfusion injury may directly affect postoperative liver function recovery and liver regeneration, which are associated with postoperative liver failure and increased mortality $(7,8)$. Thus, prevention or reduction of liver ischemia-reperfusion injury during surgery has important clinical significance for patients undergoing liver cancer surgery.

Establishing procedures to prevent or reduce hepatic ischemia-reperfusion injury has become a research focus for researchers and clinicians over the world. It was reported that administration of an appropriate anesthetic drug during surgery reduced liver reperfusion injury to a certain extent by improving liver tolerance to ischemia $(9,10)$.

Remifentanil is a potent opioid receptor agonist characterized by fast onset and rapid metabolism. It is rapidly metabolized to an inactive metabolite by plasma and tissue esterases, which is independent of the liver and kidney, thus it has been widely used in liver surgery anesthesia (11). In addition, it was recently reported that remifentanil plays a role in reducing ischemia-reperfusion injury (12). In this control study, it was found that patients receiving remifentanil during liver surgery experienced a smaller increase in the levels of the liver function markers on the first, third, and fifth day after surgery, compared with patients in the control group. Moreover, the experimental group (administered remifentanil) was better than the control group (administered isoflurane) in terms of recovery time of spontaneous breathing, time of eye opening, extubation time, and level of consciousness. This showed that administration of remifentanil during surgery reduced liver ischemia-reperfusion injury, played a protective role in postoperative liver function, and to some extent promoted recovery of liver function. In addition, remifentanil was obviously advantageous for postoperative recovery.

ICAM-1 is a single-chain transmembrane glycoprotein with a core peptide molecular weight of $55 \mathrm{kDa}$. It is a member of the immunoglobulin superfamily. When inflammation occurs, inflammatory factors are released, which can induce endothelial cells and other cells to express ICAM-1 on the cell surface, thus enhancing surface adhesion. In the present study, it was found that the level of ICAM-1 in the experimental group was lower than that in the control group, and the difference was statistically significant. This showed that the administration of remifentanil reduced the release of certain cytokines and proinflammatory factors in liver ischemia-reperfusion, resulting in a reduced inflammatory response of the liver, and protected liver cells to some extent.

Most patients with liver cancer are middle-aged and older, who show age-related low responses in autonomic reflexes, and have poor ability to adjust their response to various stimuli such as cutting, traction, and clipping during surgery. Therefore, these patients tend to experience large fluctuations in hemodynamics, and it is difficult to maintain a stable state. For older patients whose hemodynamics are unstable during surgery, selecting an appropriate anesthesia is highly important for reducing anesthesia complications (13). In the present study, it was found that when using remifentanil anesthesia during surgery, patients experienced smaller fluctuations in blood pressure than patients in the control group. This showed that remifentanil can better maintain blood pressure 
stability when patients are sedated during surgery, and thus the stability of the circulatory system.

In conclusion, remifentanil can better maintain blood pressure stability of older patients during surgery, and the stability of the circulatory system. In addition, remifentanil can reduce the release of liver-related cell adhesion molecules and inflammatory factors, thus reducing liver ischemia-reperfusion injury to protect liver function.

\section{References}

1. Kato R, Ross S and Foëx P: Fentanyl protects the heart against ischaemic injury via opioid receptors, adenosine A1 receptors and KATP channel linked mechanisms in rats. Br J Anaesth 84: 204-214, 2000

2. Yamanouchi K, Yanaga K, Okudaira S, Eguchi S, Furui J and Kanematsu T: [D-Ala2, D-Leu5] enkephalin (DADLE) protects liver against ischemia-reperfusion injury in the rat. J Surg Res 114: 72-77, 2003.

3. Parkin DM, Bray F, Ferlay J and Pisani P: Global cancer statistics, 2002. CA Cancer J Clin 55: 74-108, 2005.

4. Parkin DM, Bray F, Ferlay J and Pisani P: Estimating the world cancer burden: Globocan 2000. Int J Cancer 94: 153-156, 2001.

5. Thomas MB, O'Beime JP, Furuse J, Chan AT, Abou-Alfa G and Johnson P: Systemic therapy for hepatocellular carcinoma: Cytotoxic chemotherapy, targeted therapy and immunotherapy. Ann Surg Oncol 15: 1008-1014, 2008.

6. de Boer MT, Molenaar IQ and Porte RJ: Impact of blood loss on outcome after liver resection. Dig Surg 24: 259-264, 2007.
7. Clavien PA, Selzner M, Rüdiger HA, Graf R, Kadry Z, Rousson $\mathrm{V}$ and Jochum W: A prospective randomized study in 100 consecutive patients undergoing major liver resection with versus without ischemic preconditioning. Ann Surg 238: 843-850, discussion 851-852, 2003.

8. Banga NR, Homer-Vanniasinkam S, Graham A, Al-Mukhtar A, White SA and Prasad KR: Ischaemic preconditioning in transplantation and major resection of the liver. Br J Surg 92: 528-538, 2005.

9. Müller C, Dünschede F, Koch E, Vollmar AM and Kiemer AK: Alpha-lipoic acid preconditioning reduces ischemia-reperfusion injury of the rat liver via the PI3-kinase/Akt pathway. Am J Physiol Gastrointest Liver Physiol 285: G769-G778, 2003.

10. Bedirli N, Ofluoglu E, Kerem M, Utebey G, Alper M, Yilmazer D, Bedirli A, Ozlu O and Pasaoglu H: Hepatic energy metabolism and the differential protective effects of sevoflurane and isoflurane anesthesia in a rat hepatic ischemia-reperfusion injury model. Anesth Analg 106: 830-837, 2008.

11. Lötsch J: Pharmacokinetic-pharmacodynamic modeling of opioids. J Pain Symptom Manag 29: S90-S103, 2005.

12. Kim HS, Cho JE, Hong SW, Kim SO, Shim JK and Kwak YL: Remifentanil protects myocardium through activation of anti-apoptotic pathways of survival in ischemia-reperfused rat heart. Physiol Res 59: 347-356, 2010.

13. Kim TY, Kim DK, Yoon TG, Lim JA, Woo NS, Chee HK, Shin JK, Song MG and Kim SH: Myocardial injury in remifentanil-based anaesthesia for off-pump coronary artery bypass surgery: An equipotent dose of sevoflurane versus propofol. Anaesth Intensive Care 39: 418-425, 2011. 